\title{
DETERMINANTS OF PROGRESSION AND EQUILIBRIUM IN HYDRONEPHROSIS
}

\author{
STEPHEN A. KOFF, M.D.
}

From the Section of Urology, Department of Surgery,

University of Michigan Medical Center, Ann Arbor, Michigan

\begin{abstract}
Upper urinary tract urodynamic parameters were measured serially in 12 dogs subjected to partial ureteropelvic junction obstruction. Measurements of pressure-volume relationships (pelvimetrics) and the physiologic capacity of the renal pelvis were important predictors of hydronephrotic equilibrium which occurred only in kidneys whose pelvic urine volume remained lower than the renal pelvic capacity. Because of acquired changes in compliance and renal function, the same degree of obstruction caused progressive hydronephrosis at low pelvic volumes but not at high volumes in some kidneys. Hydronephrosis can thus be viewed as a compensatory mechanism which protects the kidney against further dilation and elevated intrapelvic pressure.
\end{abstract}

Although it generally has been assumed that hydronephrosis caused by partial ureteropelvic junction obstruction is invariably progressive, such has never been proved experimentally, and recent evidence indicates that it may not be true clinically. ${ }^{1}$ To study this problem, we have utilized a canine model with incomplete ligation of the ureteropelvic junction to produce chronic upper urinary tract dilation. Herein we examine the fate of these hydronephrotic kidneys

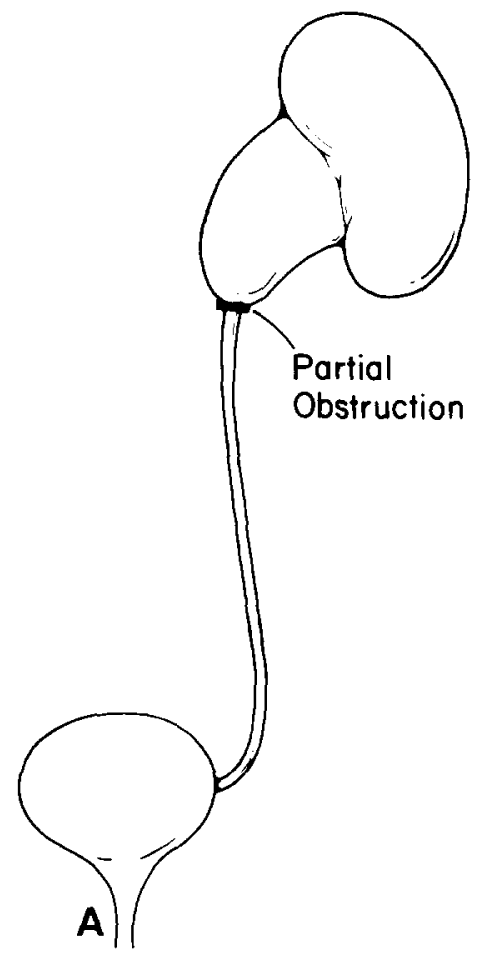

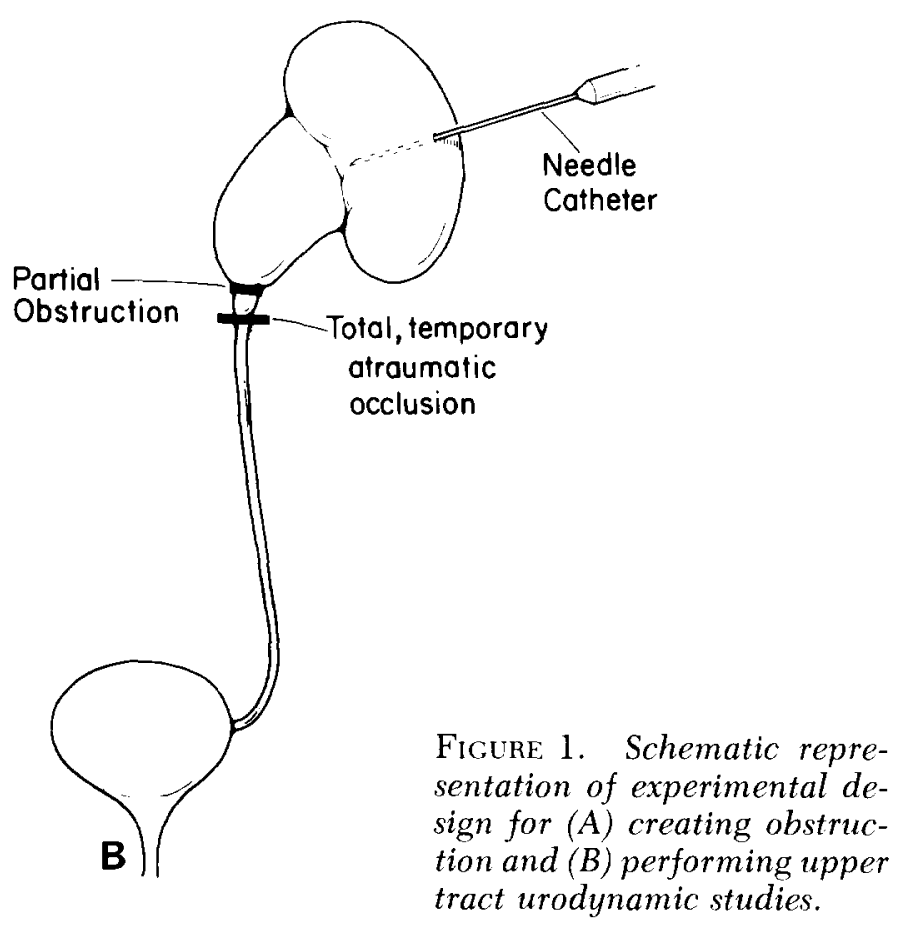

tract urodynamic studies. 
and use urodynamic techniques to determine the factors responsible for their progression or equilibration.

\section{Material and Methods}

Incomplete ligation of the ureteropelvic junction was performed in 12 adult female mongrel dogs weighing 15 to $25 \mathrm{Kg}$ under intravenous sodium pentobarbital anesthesia. The ureteropelvic junction was isolated transperitoneally and partially occluded with a 2-0 silk ligature. An 18-gauge needle was placed alongside the ureter during ligation and thereafter removed to insure that the ligation was incomplete (Fig. 1A). The animals were studied postoperatively at approximately two-week intervals as described previously. ${ }^{2}$

Resting intrapelvic pressure (IPP), was measured in all dogs under a similar state of antidiuresis using a 16-gauge Silastic needle catheter connected via a Hewlett-Packard membrane transducer and recorder to a Harvard pump which perfused saline at $1 \mathrm{ml} / \mathrm{min}$. During saline perfusion the needle catheter was thrust into the renal pelvis through the renal parenchyma to prevent leakage. The IPP was recorded immediately or after a small amount of urine which backfilled the catheter was replaced into the pelvis. After measurement of IPP, all urine contained within the kidney and renal pelvis was aspirated through the needle catheter and the volume recorded as the renal pelvic volume.

Pressure volume studies (pelvimetrics) were performed by first totally and atraumatically occluding the ureter distal to the ureteropelvic junction with a wrapped soft rubber drain and then perfusing saline through the needle catheter at a rate of $10 \mathrm{ml} / \mathrm{min}$. with the Harvard pump (Fig. 1B). Fluid thus was prevented from escaping down the ureter, and the infusion pressure was monitored and recorded. A corrected value for intrapelvic pressure was obtained after subtracting intra-abdominal pressure and the measured internal resistance of the infusion system. Whenever peristalsis occurred, the baseline pressure between contractions was used. Pressure and volume were then recorded graphically during incremental renal pelvis filling. On completion, the pelvis was emptied and the procedure repeated for accuracy.

\section{Results}

The application of a standardized partial ureteropelvic junction obstruction effectively

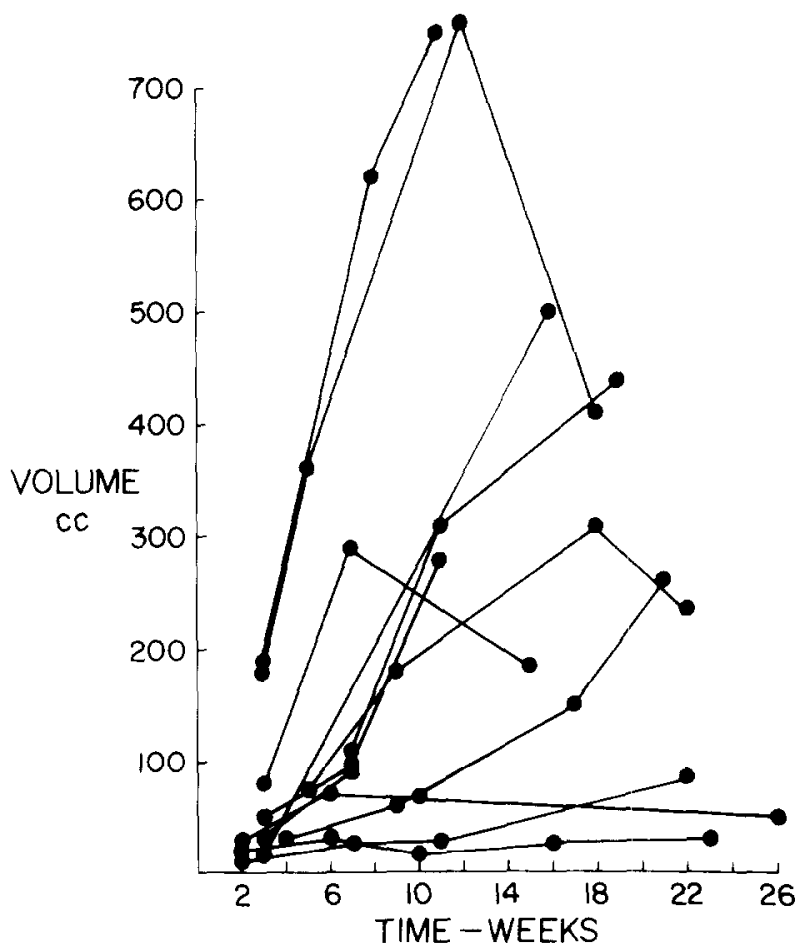

FIgURE 2. Renal pelvic volume after partial ureteropelvic junction obstruction.

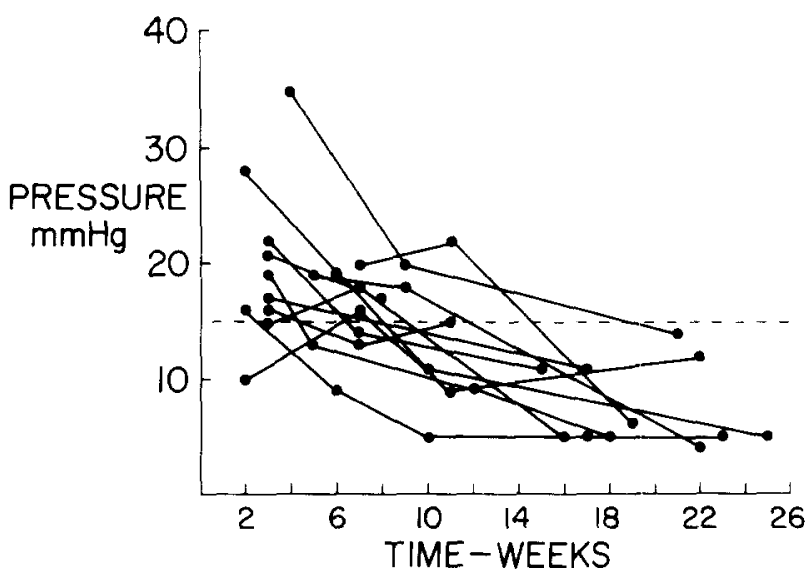

FIGURE 3. Intrapelvic pressure after partial ureteropelvic junction obstruction.

produced progressive hydronephrosis in all animals. The maximum volume of urine contained within the renal pelvic systems ranged between 27 and 1,010 $\mathrm{cc}$ as compared with the normal canine renal pelvis which contains 3 to $5 \mathrm{ml}$. Figure 2 indicates that hydronephrosis developed at different rates and that two groups of animals were definable. In the first group (3 kidneys), renal pelvic volume increased initially and then equilibrated at volumes of less than $100 \mathrm{ml}$ (range $27-88 \mathrm{cc}$, average $55 \mathrm{cc}$ ). In the 


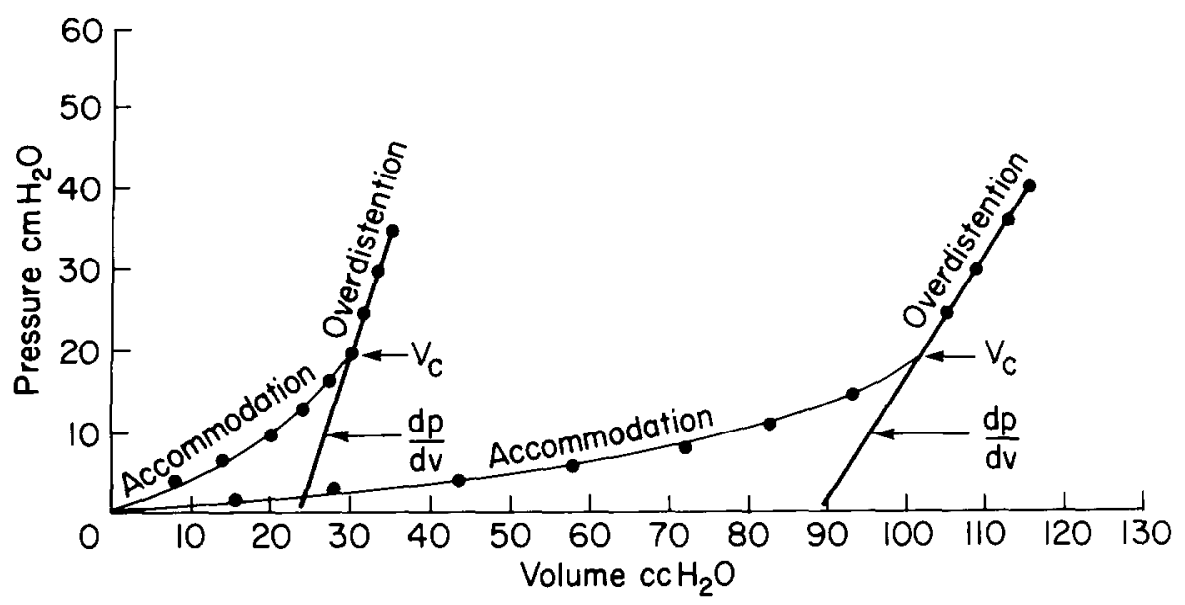

Figure 4. Pressure-volume relationships during pelvimetrics at two states in hydronephrotic dilation. (Reprinted from Koff ${ }^{2}$.)

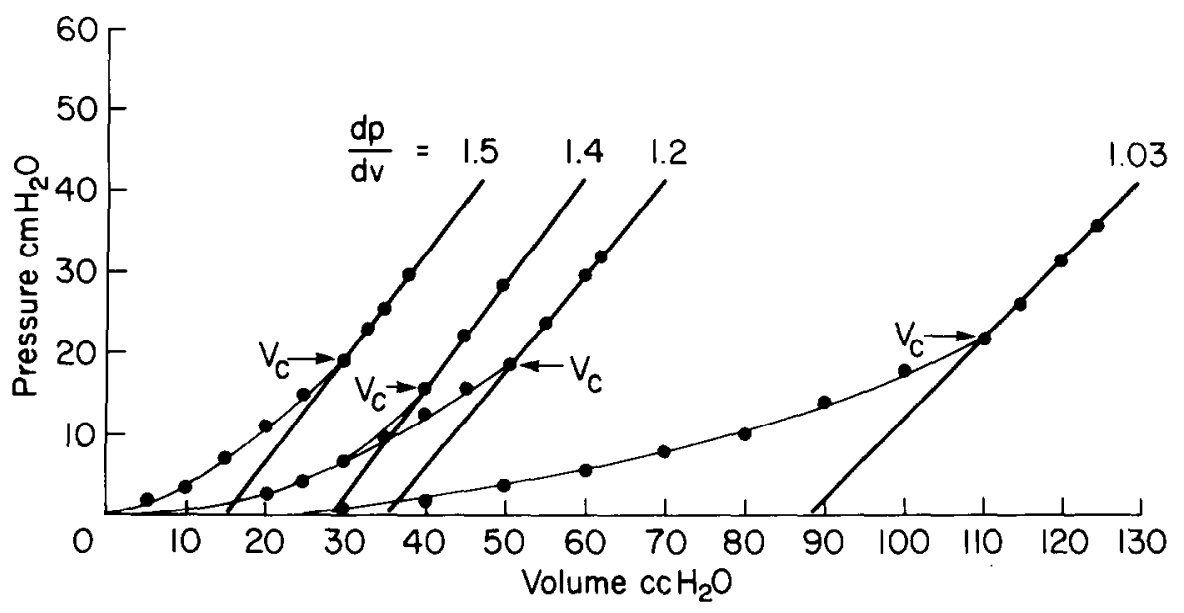

Figure 5. Sequential pressure-volume recordings from progressively enlarging hydronephrotic canine kidney illustrate increasing renal pelvic capacity and decreasing slope $(d p / d v)$ of overdistention phase. (Reprinted from Koff.)

second group, progressive hydronephrotic dilation continued, and final volumes ranged between 90 and $1,010 \mathrm{cc}$ (average $422 \mathrm{cc}$ ). In some cases, after becoming a hydronephrotic shell, the measured intrapelvic volume decreased.

During the first twelve weeks of the study, intrapelvic pressure tended to fluctuate erratically and was noted to be above the upper limit of normal value of $15 \mathrm{~mm} \mathrm{Hg}$ in many kidneys. There was no definite trend in pressure change during this time period. After twelve weeks, however, pressures tended to decrease and to normalize in all animals (Fig. 3).

Pressure-volume relationships in these progressively dilating renal pelves were examined and found to be identical to those described previously. ${ }^{2}$ All renal pelves displayed a similar pressure-volume curve composed of an accommodation limb and an overdistention limb (Fig. 4). Accommodation was characterized by a relatively flat curve in which pressure increased gradually during volume expansion. Overdis- tention occurred when the relationship between increasing volume and pressure changed and became fixed and linear. The slope of the linear overdistention portion of the curve, $\mathrm{dp} / \mathrm{dv}$, described the rate of change of pressure per unit volume. The renal pelvic volume at which overdistention occurred was designated $V_{c}$ and was defined as the physiologic capacity of the renal pelvis. Figure 5 illustrates sequential pressure volume tracings from a progressively enlarging hydronephrotic canine kidney. The slope of each curve $(d p / d v)$ during the overdistention phase is indicated as is the renal pelvic capacity at which overdistention occurred. An increasing renal pelvic capacity and a decreasing slope in the overdistention phase of the pelvimetric curve occurred during progressive hydronephrotic enlargement in all kidneys.

Intrapelvic pressure was observed to depend primarily on the degree of distention of the renal pelvis as determined by the relationship between $V_{c}$, the renal pelvic capacity and IPV, the 


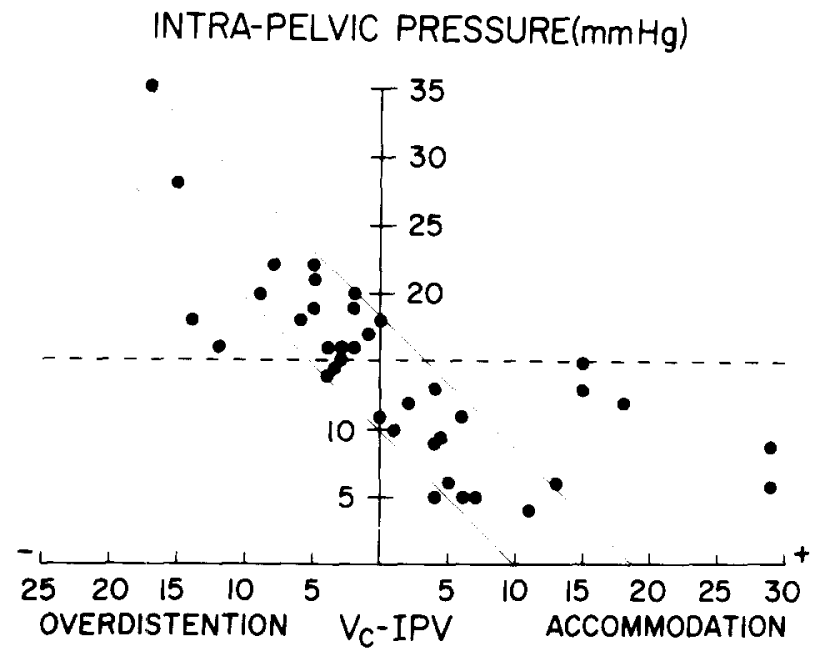

Figure 6. Comparison of intrapelvic pressure with the difference between pelvic capacity and volume (in milliliters).

renal pelvic volume (Fig. 6). Whenever the measured renal pelvic volume was less than the capacity of the renal pelvis and therefore the renal pelvis was not overdistended, $V_{c}$-IPV had a positive value and IPP was always normal, $\leq$ $15 \mathrm{~mm} \mathrm{Hg}$. On the other hand in those instances when IPV exceeded $V_{c}$ and the pelvis was overdistended, IPP was in most instances elevated above normal.

\section{Comment}

Partial ligation of the canine ureteropelvic junction proved to be a reliable model for studying chronic upper urinary tract dilation, and all kidneys became hydronephrotic with maximum pelvic volumes ranging between 27 and $1,010 \mathrm{ml}$. Figure 2 shows that within the spectrum of hydronephrotic dilation two groups of kidneys were identifiable. In the first group, the maximum volumes appeared to reach a state of equilibrium below $100 \mathrm{ml}$. Grossly, these kidneys appeared to have thick parenchyma (greater than $0.8 \mathrm{~cm}$ ) and to be functional. In the second group, dilation continued progressively until a state of massive hydronephrosis existed with the kidneys appearing as hydronephrotic shells having thin parenchyma (less than $0.3 \mathrm{~cm}$ ).

Measurements of intrapelvic pressure fluctuated widely during the first three months after ureteropelvic obstruction. This coincided with the development of hydronephrosis in all animals. After three months normal intrapelvic pressures appeared to develop in all kidneys regardless of whether they progressively dilated or reached an equilibrium. Therefore, intrapelvic pressures or changes in pressure did not distinguish kidneys which were destined to become hydronephrotic shells from those which reached an equilibrium (Fig. 3).

Pelvimetry, ${ }^{2.3}$ an experimental method for measuring the visceroelastic properties of the renal pelvis, provided useful clues as to the determinants of progression and equilibrium in hydronephrosis. It is performed by temporarily obstructing the upper ureter and then infusing fluid into the renal pelvis while measuring and recording pressure-volume relationships. The resulting curve depicts the visceroelastic properties of the renal pelvis and is similar to a cystometrogram. At first inspection, the pelvimetric curves of all dilated kidneys appeared similar in general shape as has been described previously. ${ }^{2}$ Hydronephrotic canine kidneys characteristically display accommodation or low pressure filling of the renal pelvis until the physiologic capacity of the pelvis is exceeded. Filling the pelvis above this volume results in a much more rapidly rising pressure-volume curve and the development (in most instances) of high intrapelvic pressures. The capacity of the renal pelvis is analogous to the capacity of the urinary bladder as measured by cystometry and represents the volume above which overdistention occurs due to overstretching of the visceroelastic tissue components. ${ }^{2}$

Although superficially similar in shape, the pressure-volume relationships in enlarging hydronephrotic kidneys are not identical; and we observed as the renal pelvis enlarges, its compliance changes consequent to being overstretched and thinned. This is reflected in serial measurements during dilation as an increase in the renal pelvic capacity and a decrease in the slope of the overdistention limb of the pelvimetric curve. These phenomena, which have been previously observed, ${ }^{2}$ indicate that large kidneys can accept a larger volume of fluid before becoming overdistended, and once overdistended their pressures rise at a slower rate than in small kidneys. This explains why the smallcapacity pelvis may develop very high intrapelvic pressures on being minimally overstretched whereas the large-capacity pelvis may actually undergo progressive overstretching and enlargement at pressures which are within the normal range.

Previous studies on intrapelvic pressure measurements during hydronephrosis have suggested that at any point in time, pressure was 


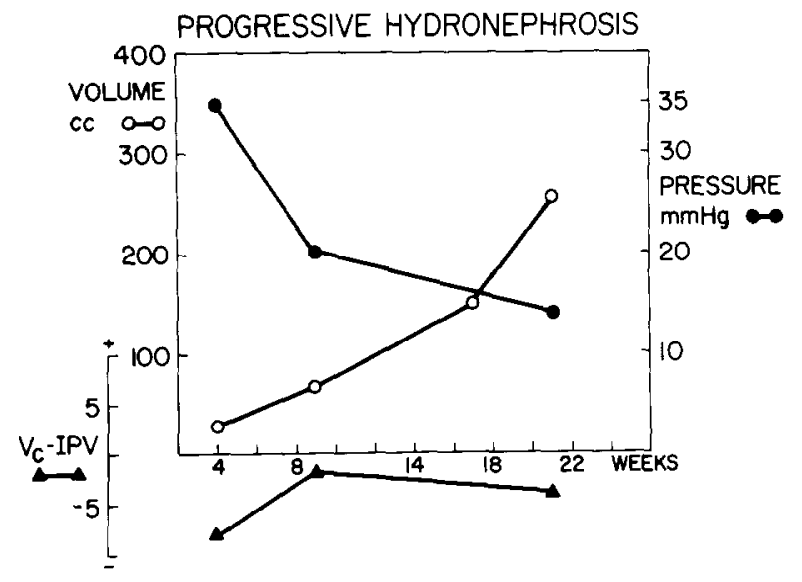

Figure 7. Time course of upper urinary tract urodynamic parameters in progressively hydronephrotic canine kidneys.

determined by the renal pelvic volume and by the degree of overdistention of the renal pelvis. ${ }^{2}$ The present data confirm this hypothesis by demonstrating that whenever pelvic volume exceeded capacity, pressures were above normal, whereas pressures were within the normal range whenever pelvic volume was less than capacity (Fig. 6). These findings establish the urodynamic significance of the pelvimetric tracing in estimating the potential for progression of hydronephrosis, when the relationship between intrapelvic volume and pelvic capacity is considered.

It is apparent from the data that the fate of all partially obstructed kidneys is not invariably progressive hydronephrosis, indicating that other factors in addition to obstruction are significant determinants of progression. These other factors include compliance and renal function. Their relationship is illustrated in Figures 7 and 8 which compare the time course of upper tract urodynamic parameters in typical examples of canine kidneys undergoing hydronephrotic progression or equilibrium. Progressive hydronephrosis occurred in only those kidneys in which the urine volumes within the renal pelvis were noted at times to be measurably greater than the pelvic capacity thereby causing progressive overdistention (Fig. 7). Interestingly, at larger pelvic volumes the intrapelvic pressures accompanying overdistention were within the normal range due to al-

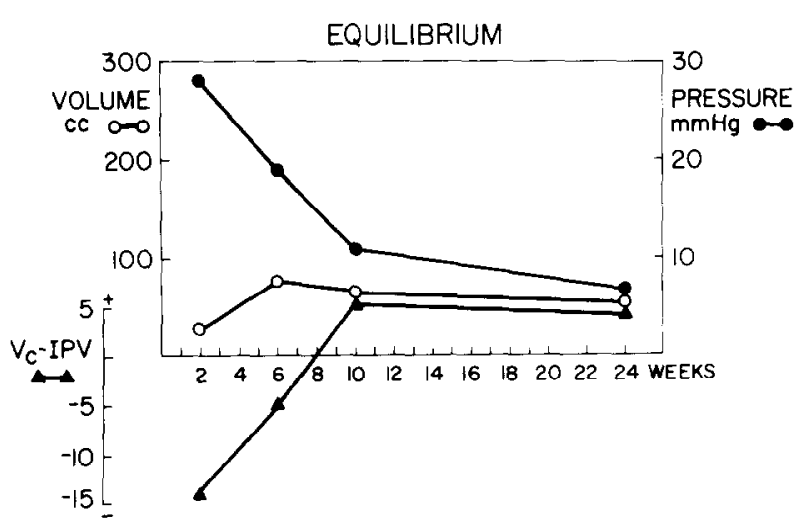

Figune 8. Time course of upper tract urodynamic parameters in canine kidney which reaches hydronephrotic equilibrium.

terations in compliance. In contrast, kidneys which reached an equilibrium displayed pelvic volumes greater than capacity only during the initial stages of hydronephrosis, but thereafter the pelvic volumes remained less than capacity and the pelves stopped expanding (Fig. 8).

These findings indicate that the same degree of partial ureteropelvic junction obstruction can cause progressive hydronephrosis in some kidneys at low pelvic volumes but not at high volumes. Hydronephrosis can thus be viewed as a compensatory mechanism which protects the kidney from overstretching its renal pelvis, high intrapelvic pressures, and progressive renal deterioration by virtue of changes in compliance that limit the rate of pressure rise and changes in renal function that limit the rate of urine formation needed to overfill the renal pelvis. ${ }^{2}$

Children's Hospital
700 Children's Drive
Room C 304
Tolumbus, Ohio 43205

\section{References}

1. Koff SA: Clinical management of patients with idiopathic hydronephrosis, in O'Reilly P, and Gosling J (Eds): Idiopathic Hydronephrosis, Berlin: Springer-Verlag, 1981, pp 115-130.

2. IDEM: The diagnosis of obstruction in experimental hydronephrosis. Mechanisms for progressive urinary tract dilation, Invest Urol 19: 85 (1981).

3. Olsen PR: The renal pelvis and ureteral peristalsis. I. Pelevometry, Scand J Urol Nephrol 13: 269 (1979). 